\title{
Landmark analysis: A primer
}

\author{
Charity J. Morgan, PhD \\ a Department of Biostatistics, University of Alabama at Birmingham, Birmingham, AL, UK
}

Received Jan 2, 2019; accepted Jan 2, 2019

doi: 10.1007/s12350-019-01624-z

In the survival analysis setting, landmark analysis refers to the practice of designating a time point occurring during the follow-up period (known as the landmark time) and analyzing only those subjects who have survived until the landmark time. ${ }^{1}$ A comprehensive overview of the landmark analysis method and its use has been provided by Dafni. ${ }^{2}$ To understand why landmark analysis is sometimes necessary, consider the following hypothetical example.

A study is conducted in which all subjects were treated at baseline and then followed for a period of several years. During follow-up, a subset of subjects were found to respond favorably to treatment, with response times varying among responders.

Figure 1 displays the response times for three hypothetical subjects during the first six months of the study. The dashed lines represent the time during which the subject has not responded to treatment; for subjects that respond to treatment, solid lines are used to denote the time during which the subject has responded. After six months, Subject 1 has not responded. Subjects 2 and 3 responded to treatment after 1.6 and 2.9 months, respectively.

Study investigators might be interested in determining whether the responders had a significantly longer survival times than non-responders. A naive (and incorrect) approach would be to classify Subject 1 as a non-responder and Subjects 2 and 3 as responders and then use standard survival analysis methods, such as Kaplan-Meier estimation or Cox proportional hazard modeling, to compare the survival of these two groups following treatment. Such an approach effectively assumes that Subjects 2 and 3 responded to treatment immediately. However, at baseline (i.e., the time of treatment), responder status is unknown for all subjects.

\footnotetext{
Reprint requests: Charity J. Morgan, PhD, Department of Biostatistics, University of Alabama at Birmingham, 1720 Second Avenue South, Birmingham, AL 35294-0022, UK; cjmorgan@uab.edu

J Nucl Cardiol 2019;26:391-3.

$1071-3581 / \$ 34.00$

Copyright (C) 2019 American Society of Nuclear Cardiology.
}

That is, Subjects 2 and 3 begin the study as non-responders and do not become responders until later in follow-up. Assuming otherwise ignores the fact that responders, by definition, are guaranteed to survive at least until the time of response; this error is known as the guarantee-time bias or the immortal time bias. ${ }^{3,4}$

Landmark analysis may be used to avoid this type of bias. Under this approach, a landmark time is selected and any subjects who were lost to follow-up or died prior to this time are excluded from further analysis. For the remaining subjects, subjects who have responded by the landmark time are classified as responders. Any subject who has not responded by the landmark time is classified as a non-responder, even if that subject later responds to treatment.

Figure 2 provides two examples of this process. In Figure 2A, a landmark time of two months post-treatment is chosen. Subjects 1 and 2 are then classified as non-responders because neither subject has responded to treatment at that time. Note that even though Subject 2 will later go on to respond to treatment, to avoid the guarantee-time bias, Subject 2 must be considered to be a non-responder due to his or her status at the landmark time. Subject 3 responded to treatment prior to the landmark time and is thus classified as a responder. As can be seen in Figure 2B, selecting a different landmark time (here, four months post-treatment) may change the classification of some subjects. In this example, the later landmark allows Subject 2 enough time to respond to treatment, leading to this subject being placed in the responder group.

Once the landmark has been chosen, any ineligible subjects have been excluded, and subjects have been classified according to their status at the landmark time, the usual survival analysis methods are applied. The results of these methods are interpreted as usual, with the important caveat that conclusions are only generalizable to subjects who have survived until the landmark time.

While landmark analysis was originally proposed to evaluate the association between treatment response and survival, ${ }^{1}$ or to allow for groupings based on any 


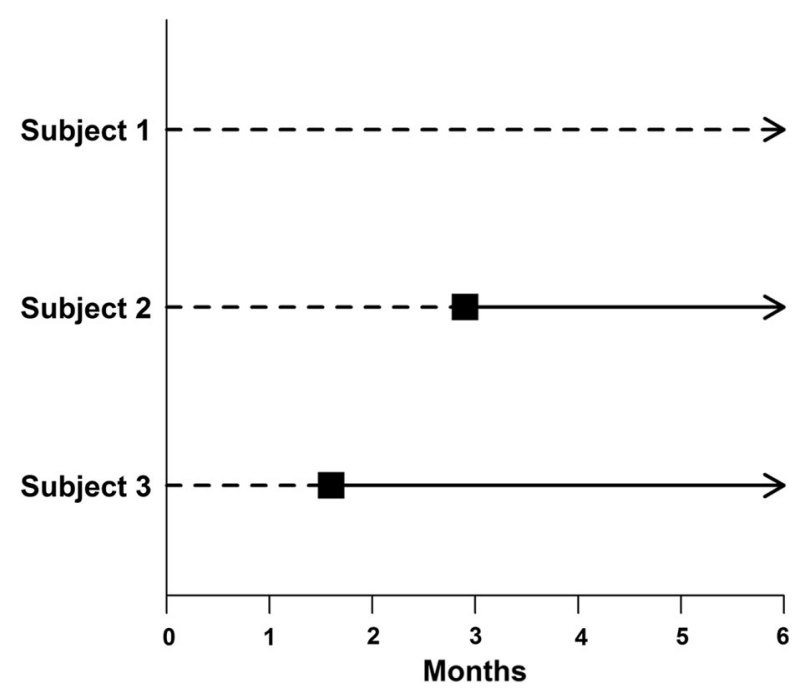

Figure 1. Response times for three hypothetical subjects. Time of response indicated with a square. Dashed lines represent time the subject has not responded to treatment; solid lines denote the time during which the subject has responded.

covariate whose value is not known at baseline, this method does not necessarily need to be used for a group comparison. In some cases, it may be of interest to simply compare the survival experience both before and after the landmark (see Figure 3).

Results can vary according to the landmark time, so the choice of landmark is a critically important consideration. Because events occurring prior to the landmark time are excluded from the analysis, choosing a later landmark will result in a smaller sample size and therefore lower statistical power. The earlier the landmark time, the more likely late responders are to be misclassified as non-responders (as with Subject 2 in Figure 2A). To avoid bias, the landmark should be chosen before data analysis begins and ideally should correspond to a clinically meaningful period of time. ${ }^{2}$ When the choice of landmark time is not obvious, the data should be analyzed using multiple landmark times in order to determine the sensitivity of the findings to the choice of landmark. ${ }^{3}$

An alternative to landmark analysis is a Cox proportional hazards model that allows the value of the covariate indicating treatment response to vary over time. ${ }^{5}$ This extension of the Cox model allows the entire follow-up period to be considered (rather than only the post-landmark time) and does not require any subjects or events to be excluded from analysis. Furthermore, $\mathrm{Dafni}^{2}$ notes that this approach would avoid both the problem of selecting a landmark time and any misclassification errors. However, interpretation of the results from these models can be difficult. ${ }^{6}$ Further discussion
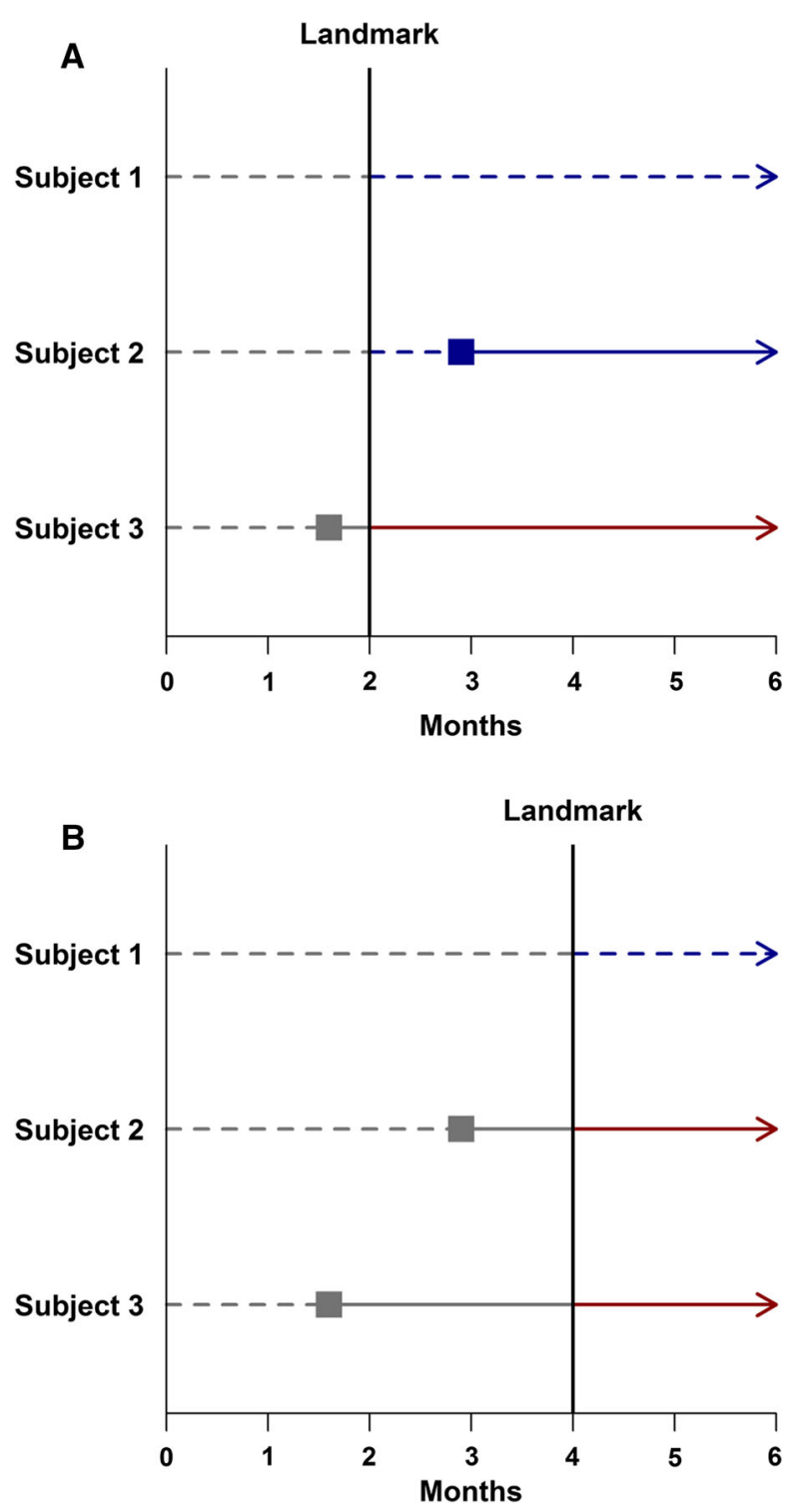

Figure 2. Classification of hypothetical subjects according to status at landmark. Subjects are classified as either responders (red) or non-responders (blue) based on their status at the landmark time.

of time-varying Cox models may be found in Fisher and Lin. ${ }^{6}$

Application of the landmark analysis method is straightforward and yields easily interpretable results. Nevertheless, the choice of landmark time must be carefully considered and sensitivity analyses should be utilized whenever there are multiple landmark times under consideration. For settings where an appropriate landmark cannot be determined or where results are overly sensitive to the choice of landmark, a Cox model with time-varying covariates provides a feasible alternative. 


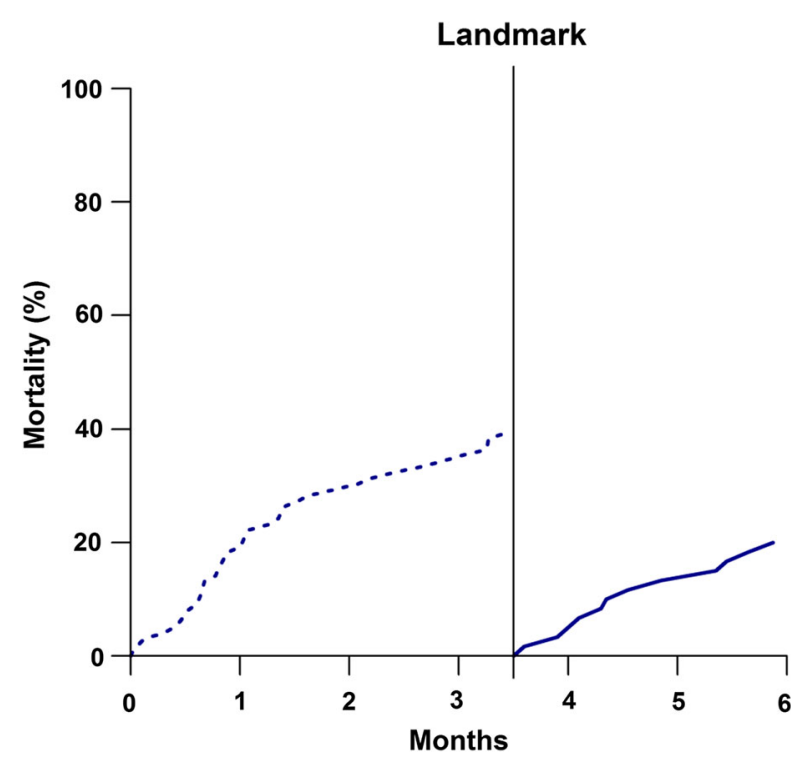

Figure 3. Kaplan-Meier estimates of survival before and after the landmark time.

\section{Disclosure}

The author has no conflicts of interest to disclose.

\section{References}

1. Anderson JR, Cain KC, Gelber RD. Analysis of survival by tumor response. J Clin Oncol 1983;1:710-9.

2. Dafni U. Landmark analysis at the 25 -year landmark point. Circ Cardiovasc Qual Outcomes 2011;4:363-71.

3. Buyse M, Piedbois P. On the relationship between response to treatment and survival time. Stat Med 1996;15:2797-812.

4. Gleiss A, Oberbauer R, Heinze G. An unjustified benefit: Immortal time bias in the analysis of time-dependent events. Transpl Int 2018;31:125-30.

5. Cox DR. Regression models and life-tables. J R Stat Soc Ser B 1972;34:187-220.

6. Fisher LD, Lin DY. Time-dependent covariates in the Cox proportional-hazards regression model. Annu Rev Public Health 1999;20:145-57.

Publisher's Note Springer Nature remains neutral with regard to jurisdictional claims in published maps and institutional affiliations. 